\title{
'Me And Pac And Snoop' + 'Variable:Posture'
}

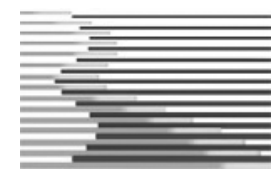

Ambrose Naqeeb Stevens

Department of Art, University of Iowa, Iowa City, IA USA

Poroi 7, 2 (June 2011)

Title: Me and Pac and Snoop

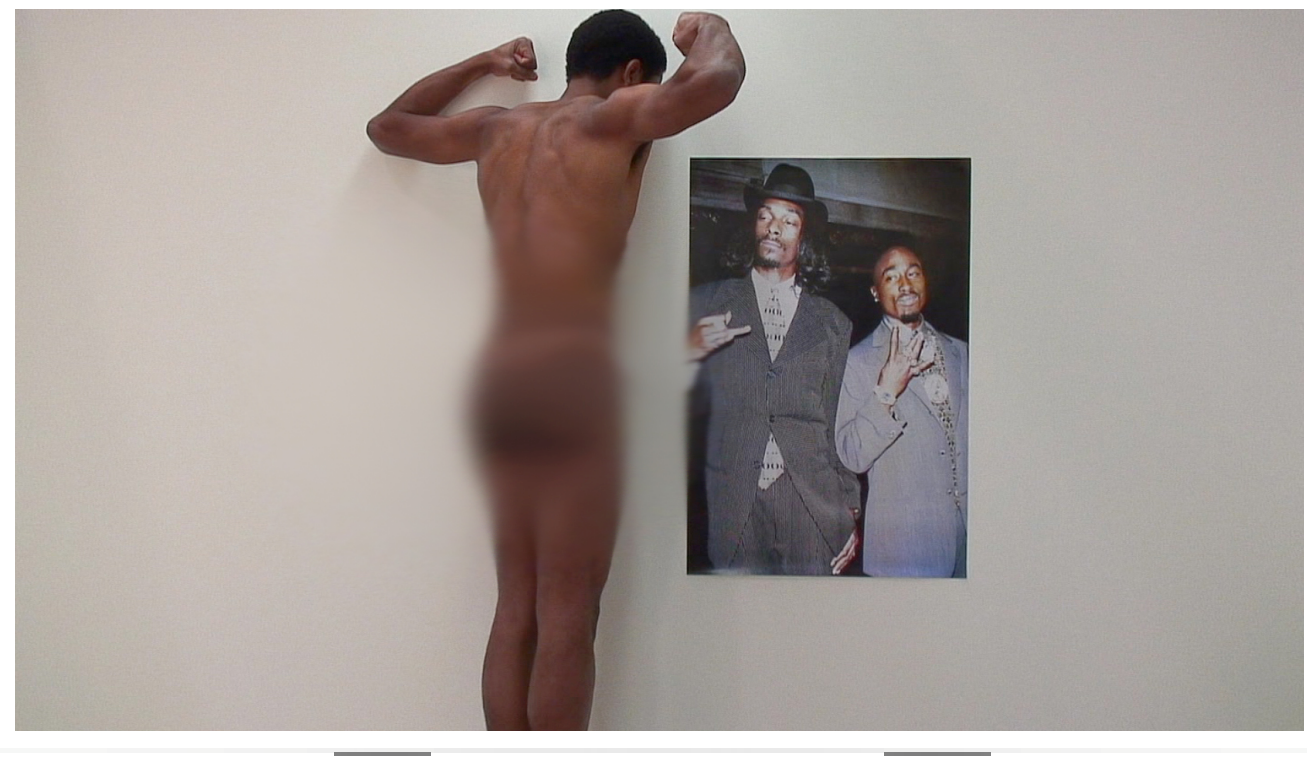

Date: 2010

Medium: Video

This work confronts constructions of masculinity in depictions of rap musicians. Having lived in Los Angeles during the earlier part of my life, these two figures played a significant role in the visual cultural landscape that I experienced. During this performance, I activate the two figures by positioning myself within their gaze. Utilizing this relationship, I attempt to maintain an upper body muscular flex for as long as possible. Once I am no longer capable of holding the flex I relax, still within their gaze. In exhausting the capacities of my body, I seek to open 
questions of reality, representation and the relationship between them.

$$
* * *
$$

\section{Title: Variable: Posture}

Date: 2010

Medium: Video

This work plays with the performance of gender. Utilizing

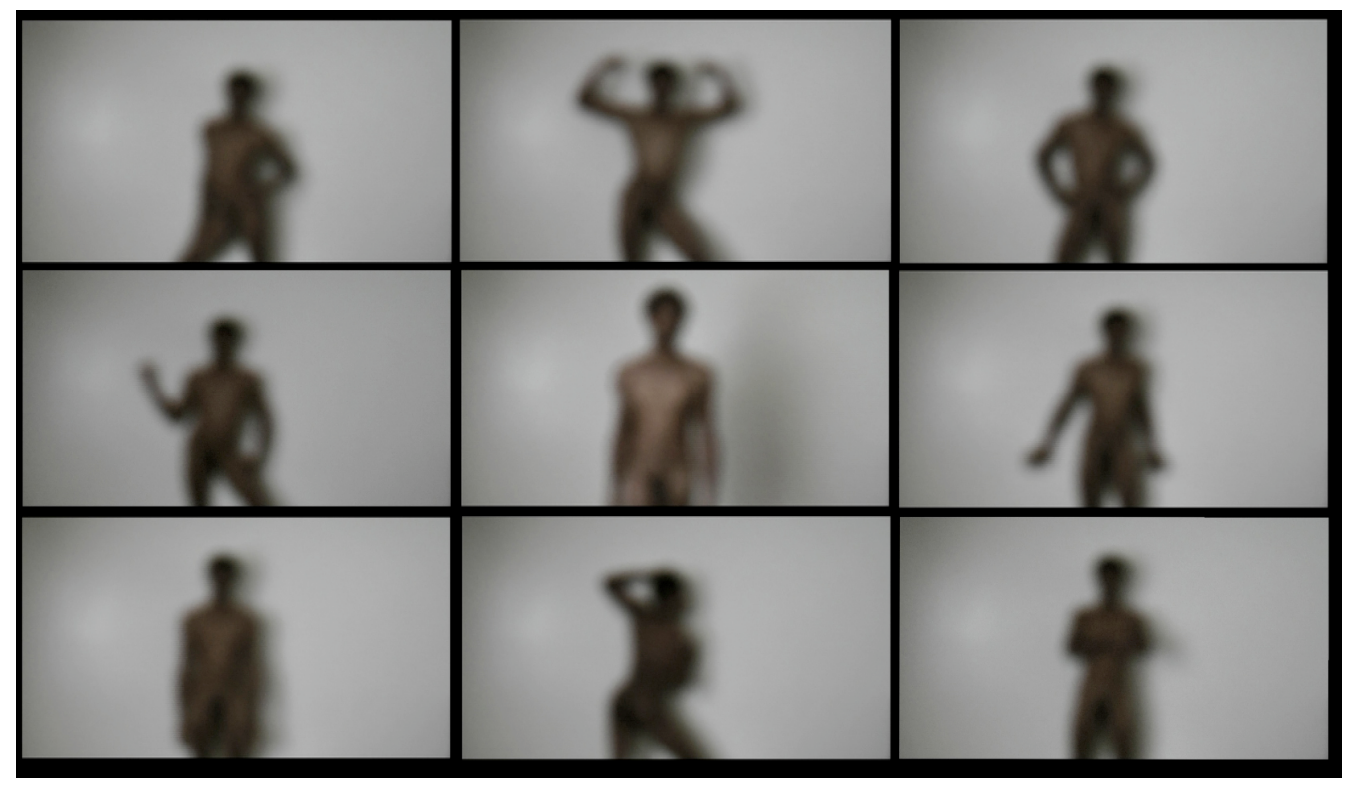

both the concept and the act of 'posturing,' in this piece I contemplate the way bodies are understood visually. Intentionally out of focus and situated in a grid, the formal decisions of the work illustrate the role of ambiguity in perceptions of gender. The figure enters each frame simultaneously, assumes a different pose, freezes for a brief moment, and then exits. 